\title{
Evaluating the Signal Processing Capacities of Post-Mortem Cerebral Cortical Tissue by Artificial Phototransduction of Dynamic Visual Stimuli
}

\author{
Nicolas Rouleau, Justin N. Costa, Michael A. Persinger \\ Biomolecular Sciences, Biology, and Behavioural Neuroscience Programs Laurentian University, Sudbury, Ontario, Canada \\ Email:ny_rouleau@laurentian.ca
}

How to cite this paper: Rouleau, N., Costa, J.N. and Persinger, M.A. (2017) Evaluating the Signal Processing Capacities of PostMortem Cerebral Cortical Tissue by Artificial Phototransduction of Dynamic Visual Stimuli Open Journal of Biophysics, 7, 113.

http://dx.doi.org/10.4236/ojbiphy.2017.71001

Received: November 8, 2016

Accepted: December 3, 2016

Published: December 6, 2016

Copyright $(\odot) 2017$ by authors and Scientific Research Publishing Inc. This work is licensed under the Creative Commons Attribution International License (CC BY 4.0).

http://creativecommons.org/licenses/by/4.0/ C) (i) Open Access

\begin{abstract}
The signal processing function of human cerebral cortical tissues is determined by the regional cytoarchitectures distributed throughout the brain. Based upon this assumption, we pursued the hypothesis that residual microstructure within the primary and associative visual cortices of a fixed, post-mortem whole human brain would process electrical signals differentially. To this end, we designed and engineered a very simple brain-photocell interface. Photostimuli, presented as either periodic flashes or as dynamic visual images, were transduced by photocells attached to the optic nerve of a post-mortem human brain specimen. The novel approach revealed that microvolt fluctuations within the primary and associative visual cortices could be discriminated. Simple light-dark discrimination was noted for the primary visual area (BA17) whereas within the right occipito-parietal cortices of the dorsal visual stream (BA19, BA7), spectral power of microvolt fluctuations could discriminate moving visual stimuli from those which were non-moving. Discriminant analysis classified movement represented within the right parietal lobe with $80 \%$ success. Together, the results suggest that artificially generated electrical signals are processed differentially by alternative cortical regions in the post-mortem brain.
\end{abstract}

\section{Keywords}

Phototransduction, Superior Parietal Cortices, Occipital Cortices, Fixed Human Brain, Quantitative Electroencephalography (QEEG)

\section{Introduction}

One of the principle assumptions of modern neuroscience is that structure dictates function. A corollary of this principle is that whole brain structure should determine large-scale function while microstructures, such as those at the level of the synapse, would be expected to determine functions at micro- and nanometer scales. If brain 
structure is maintained, function is necessarily and inseparably expressed. There is no a priori reason that some of the fundamental microstructure of the human brain that determines its specific complex functions should not be preserved to some degree in appropriately fixed human brain specimens. Rouleau et al. [1] have shown clear histopathological evidence that the general detail of the cerebral cortices of fixed human brains is still visibly evident decades after the cessation of function. Further, Rouleau \& Persinger [2] demonstrated that the right parahippocampal cortices (of coronal sections) of a brain preserved in ethanol-formalin-acetic acid for decades could be induced to express increased theta power upon discrete electrical current injections into the tissue.

The supposition that gross differential structural organization of the human brain reflected the unique or exceptional capacities and traits of the person while he or she was alive was a rationale for maintaining the cerebrums of scientists, musicians, and technically-extraordinary people in brain banks during the late 19th century. It was assumed that the discernable gross and sometimes general histological differences in specific regions were related to the person's unique behaviors while alive. This approach was evident in the motivation to carefully assess very exceptional brains such as Albert Einstein [3] [4]. The functional capacity of the human brain was not explored systematically until appropriate faradic stimulation was applied to dog [5] and human brains [6]. Direct stimulation of epileptic brains during surgery by F. Krause [7] preceded the remarkable clinical experiments by Wilder Penfield and his colleagues [8] [9] during the middle of the 20th century. These experiments demonstrated that complex experiences, forced-thought, and myriad behaviours could be elicited by stimulation of the cortical manifold. The development of sophisticated quantitative measurements such as quantitative electroencephalography (QEEG), the complex technical tools to extract information from these data, and photocells have created the conditions to explore the residual properties of appropriately fixed human brains by coupling organic tissue to electrical circuits. The discernment of these properties could be revealing with respect to the nature of the minimum amount of microstructure that is correlated with complex functions and consciousness.

Brain interfaces with electronic circuits, though typically reserved for living individuals, could be applied to post-mortem brain tissues in order to investigate signal processing capacities of the cerebral cortical tissue. Here, we present a simple brainphotocell interface which was used to activate both occipital and parietal cortices which are known to express visual functions in living subjects. These experiments represent the first ever post-mortem brain interface with an electronic circuit wherein functional signal processing at the level of the cortex can be inferred. Here we show that the primary and associative visual cortices differentially filter patterns of stimulation presented artificially through photosensors attached to the optic nerves.

\section{Material and Methods}

The purposes of the experiments were 1) to induce artificial phototransductions of pulsed light in a chemically fixed human brain while tracing the expression of frequency-dependent microvolt fluctuations across the rostro-caudal axis of the cerebrum and 2) to observe its capacity to filter multi-channel photoarray signals to discriminate dy- 
namic visual stimuli. In the first set of experiments, the left or right optic nerve was equipped with a photoresistor which transduced light pulses into waves of current which were measured at the level of the left or right occipital pole. Square wave signals, pulsed at low and high frequencies were transmitted to a flashlight which generated light patterns directed to the brain during simultaneous recording of microvolt fluctuations within the occipital poles. In the second set of experiments, a 4-photocell array converging to optic nerve inputs conveyed signals from dynamic visual stimuli emitted by a liquid crystal display (LCD) monitor. The signal processing capacities of primary receptive occipital cortices (BA17) and associative visual areas within the occipito-parietal cortices (BA7, BA19, dorsal visual stream) were assessed. Discriminations between types of dynamic stimuli were performed based upon spectral power densities derived from microvolt potential recordings of occipital and parietal cortical tissue relative to cerebral vasculature.

\subsection{Electrophysiological Measurement Device \& Spectral Power}

A Mitsar 201 amplifier, typically used in human quantitative electroencephalography (QEEG), was coupled to a series of needle electrodes which were inserted into select gyri as well as the ventral cerebral vasculature. Four electrodes were inserted directly into the basilar artery. A fifth electrode was inserted into the gyrus of interest. The average electric potential difference between the four basilar artery needle electrodes (references) and the fifth electrode served as the measurement. These values, in microvolts $(\mu \mathrm{V})$, were recorded within WinEEG (version 2.93.59) at a rate of $250 \mathrm{~Hz}$ on an $\mathrm{HP}$ ENVY laptop computer running a Windows 8 operating system. A low-cut filter of 1.5 $\mathrm{Hz}$, high-cut filter of $50 \mathrm{~Hz}$, and notch filters with $50 \mathrm{~Hz}-70 \mathrm{~Hz}$ and $110 \mathrm{~Hz}-130 \mathrm{~Hz}$ band ranges were applied in order to eliminate electrical artifacts. Data were extracted from WinEEG as raw strings and spectral analyzed within SPSS v20. Spectral power density (SPD) bins were computed for classic QEEG band ranges: Delta $(1.5 \mathrm{~Hz}-4 \mathrm{~Hz})$, theta $(4 \mathrm{~Hz}-7.5 \mathrm{~Hz})$, alpha $(7.5 \mathrm{~Hz}-14 \mathrm{~Hz})$, beta1 $(14 \mathrm{~Hz}-20 \mathrm{~Hz})$, beta2 $(20 \mathrm{~Hz}-30$ $\mathrm{Hz})$, and gamma $(30 \mathrm{~Hz}-40 \mathrm{~Hz})$.

\subsection{Brain Specimen}

A full, unsectioned human brain fixed in ethanol-formalin-acetic acid served as the specimen which received phototransduced light flash patterns. In a previous study [1], we demonstrated that this specimen expressed an intrinsic voltage gradient which was characterized by a left-rostral, right-caudal hemispheric dominance. Our measurements indicated that the resistance from the rostral to the caudal pole of the cerebrum was 35 kOhms. It displayed all of the major gyri and sulci outlined by Crosby, Humphrey \& Lauer [10] and was unremarkable on a gross structural level. The brain stem, delimited by the caudal medulla was preserved with the cerebellum affixed. The cerebrovasculature was partially preserved, with the middle (MCA), anterior (ACA), and posterior (PCA) cerebral arteries still intact. The basilar artery served as the electrical reference point-a method which had been developed over previous studies [1] [2]. For the purposes of this study, we required a partially intact visual pathway. The optic nerves were both preserved with an unsevered optic chiasm as well intact optic tracts. There was no 
indication that downstream components of the visual pathway were structurally compromised in ways unaccounted for by fixation. Other specimens available to us did not meet the aforementioned anatomical criteria and were therefore excluded from testing, limiting our sample to a single, full human brain.

\subsection{Phototransduction Apparatus}

In the first set of experiments, a single cadmium sulphide photoresistor $(2 \times 4 \times 5 \mathrm{~mm})$ with a spectral response peak of $540 \mathrm{~nm}$ coupled to three $1.5 \mathrm{~V}$ batteries (in series) was inserted into the left or right optic nerve of the fixed human brain (Figure 1). At 10 lux, the light resistance was $8-20 \mathrm{kOhm}$ whereas the dark resistance at 0 lux was $1 \mathrm{MOhm}$. The total voltage provided by the power source was $4.5 \mathrm{~V}$, where increased magnitude of light at the interface of the photoresistor generated increased conduction (decreased resistance) of electric current. The circuit, which is connected to the optic nerve, served as an artificial phototransduction apparatus analogous to a very low spatial resolution photoreceptive unit such as a rod cell, though relatively amplified. Measurements using a digital multimeter indicated that the resistance of the circuit was $20 \mathrm{kOhm}$ under fluorescent lights in the laboratory.

A flashlight coupled to a waveform function generator served as the stimulating device which triggered photoconduction at the level of the optic nerve. A square wave sweep of frequencies ranging between $1 \mathrm{~Hz}-7 \mathrm{~Hz}$ in increments of $1 \mathrm{~Hz}$ as well as 10 $\mathrm{Hz}-70 \mathrm{~Hz}$ in increments of $10 \mathrm{~Hz}$ were pulsed as light flashes directed to the photoresistor, which was positioned $50 \mathrm{~cm}$ away. Luxmeter measurements indicated values of 100 lux adjacent to the light source, which were attenuated to values approximating 10 lux at the level of the photoresistor.

In the second set of experiments, a 4-photocell array affixed to a foam scaffold (Figure 2(a)) served as a low-spatial-resolution artificial retina (i.e., multiple photoreceptive units). Each photoresistor in the array drew power from an independent $4.5 \mathrm{~V}$

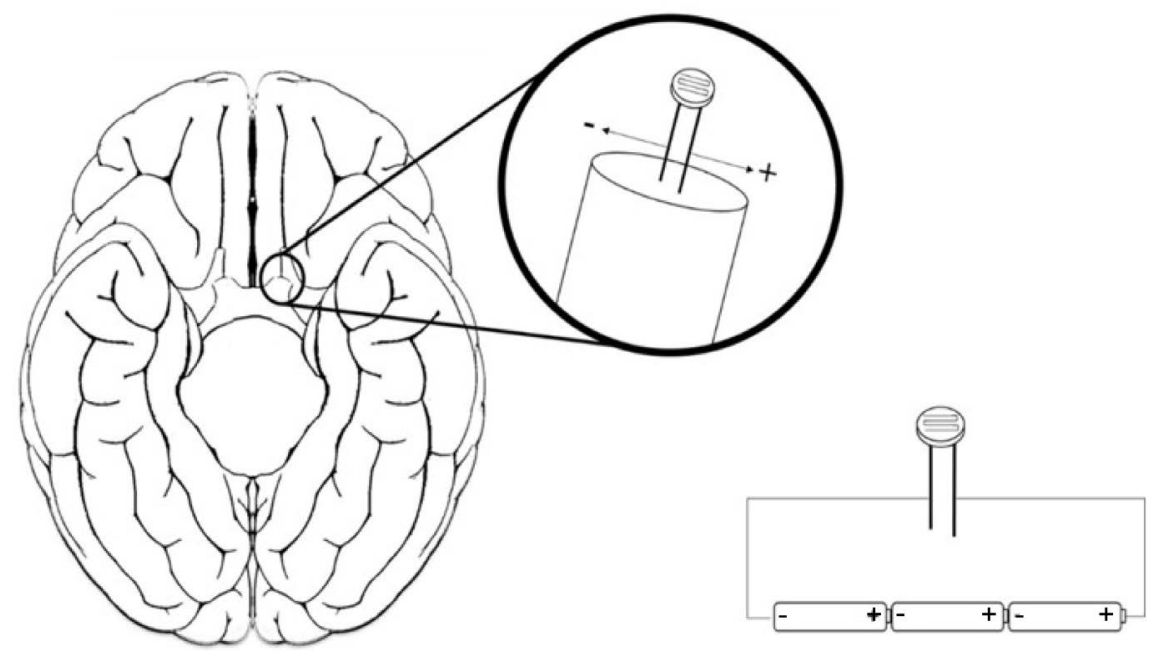

(a)

(b)

Figure 1. Ventral surface of the human brain specimen with a photoresistor inserted into the left optic nerve (a). The phototransduction circuit showing a photoresistor coupled to three $1.5 \mathrm{~V}$ AA-batteries $(\mathrm{b})$ in series ( $4.5 \mathrm{~V}$ power source) is presented as a schematic. 

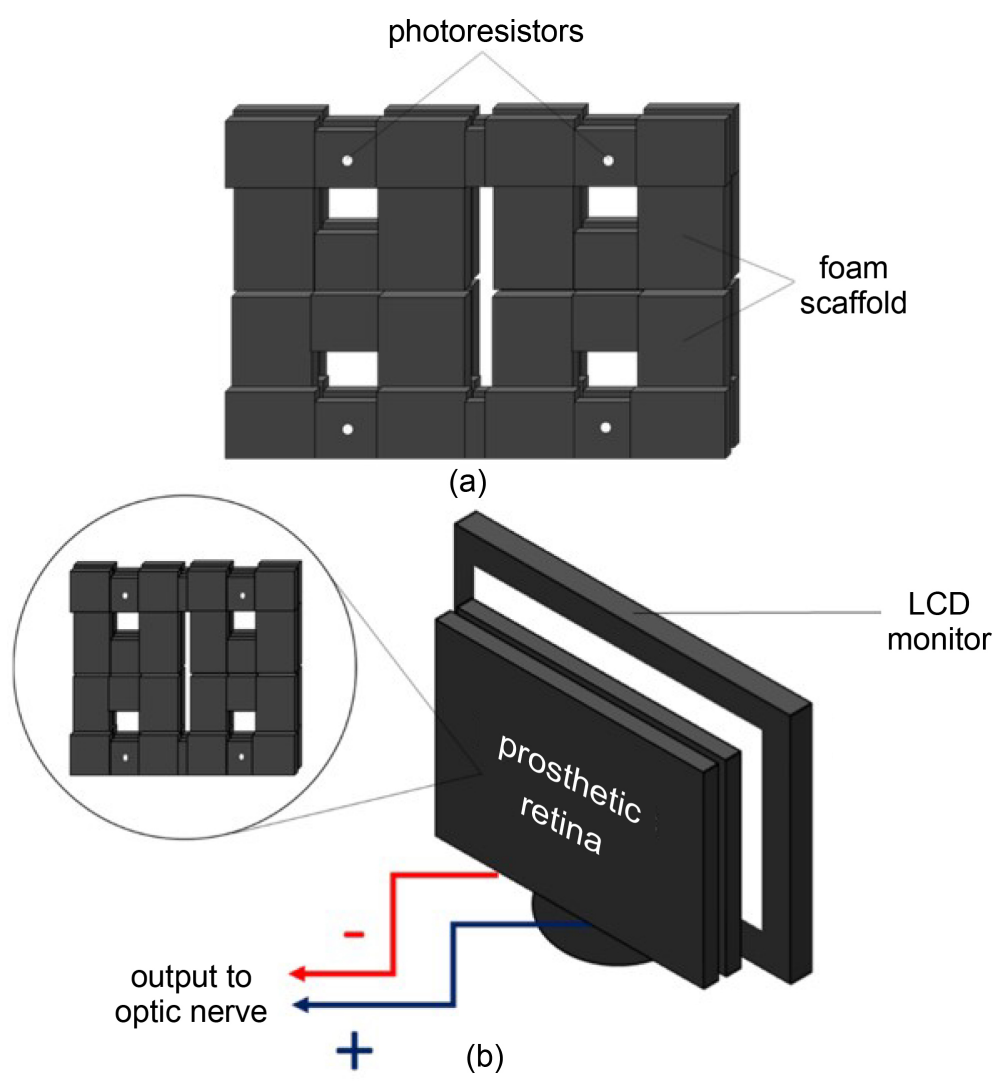

Figure 2. A 4-photocell array affixed to a foam scaffold (a) was positioned $3 \mathrm{~cm}$ in front of an LCD monitor (b). Each photoresistor in the array drew from an independent power source, converging as a single positive and negative lead (needle electrodes) which was inserted into the optic nerve of the brain.

source, converging at the optic nerve as a single positive and negative lead. A $56 \mathrm{~cm}$ (diagonal) LCD monitor (Figure 2(b)) positioned $3 \mathrm{~cm}$ away from the artificial retina displayed a series of dynamic visual images in addition to static black or white control conditions, each lasting 60 seconds.

It was reasoned that, for the purposes of public access and independent replication, stimuli should be sourced from open media platforms. Each visual stimulus, consisting of 60 seconds of a YouTube video (excluding title screens and captions) containing a common URL component (i.e., http://youtu.be/) followed by an individual URL code (e.g., XIMLoLxmTDw) will be presented here and can be combined (i.e.

https://youtu.be/XIMLoLxmTDw) to independently access the stimuli. All video conditions were muted and displayed in 480p greyscale to avoid confounds of resolution or colour biases based upon the peak wavelength of the photoresistor.

A constant strobe (4 Hz, URL: 9USauSZJp1A) and variable strobe (URL: -Nk6sCIU3NE), Mandelbrot fractals (URL: 3YzGKMR_wtg), spirals (URL: 0lkjoTh1Gpk), one moving light (URL: ynHlGP6iSbI) or many simultaneously moving lights (URL: sLe18h3PDD0), as well as potentially emotionally affective visual stimuli including adult humans dancing (URL: 0emcNq7T-aA), babies and small dogs playing together (URL: p336IIjZCl8), war footage (URL: qKqROjL14uw), and violent graphic imagery (URL: P0RACxeMjhw) were displayed. 


\section{Results}

\subsection{Rostro-Caudal Signal Transform}

Standardized spectral profiles of baseline, $10 \mathrm{~Hz}$, and $20 \mathrm{~Hz}$ stimulation conditions were plotted, revealing marked ipsilateral hemispheric increases in band-matched SPDs within the occipital pole, Fisher's $\mathrm{z}>1.96, \mathrm{p}<0.001$ (Figure 3). Right optic nerve input (Figure 3(a) and Figure 3(b)) resulted in increased band-matched z-scores for $10 \mathrm{~Hz}$ stimulation $(7.5 \mathrm{~Hz}-14 \mathrm{~Hz}$ SPD band) and $20 \mathrm{~Hz}$ stimulation $(20 \mathrm{~Hz}-30 \mathrm{~Hz}$ SPD band) within the ipsilateral occipital pole (Figure 3(b)) relative to the contralateral occipital pole (Figure 3(a)). Left optic nerve input (Figure 3(c) and Figure 3(d)) of 10 $\mathrm{Hz}$ and $20 \mathrm{~Hz}$ stimulation resulted in similar ipsilateral increases relative to the contralateral occipital pole. Whether the positive lead of the photoresistor was inserted into the nasal or temporal aspect of the optic nerve did not affect the ipsi- or contralateral expression of increased band-matched SPDs ( $p>0.05)$. However, it should be noted

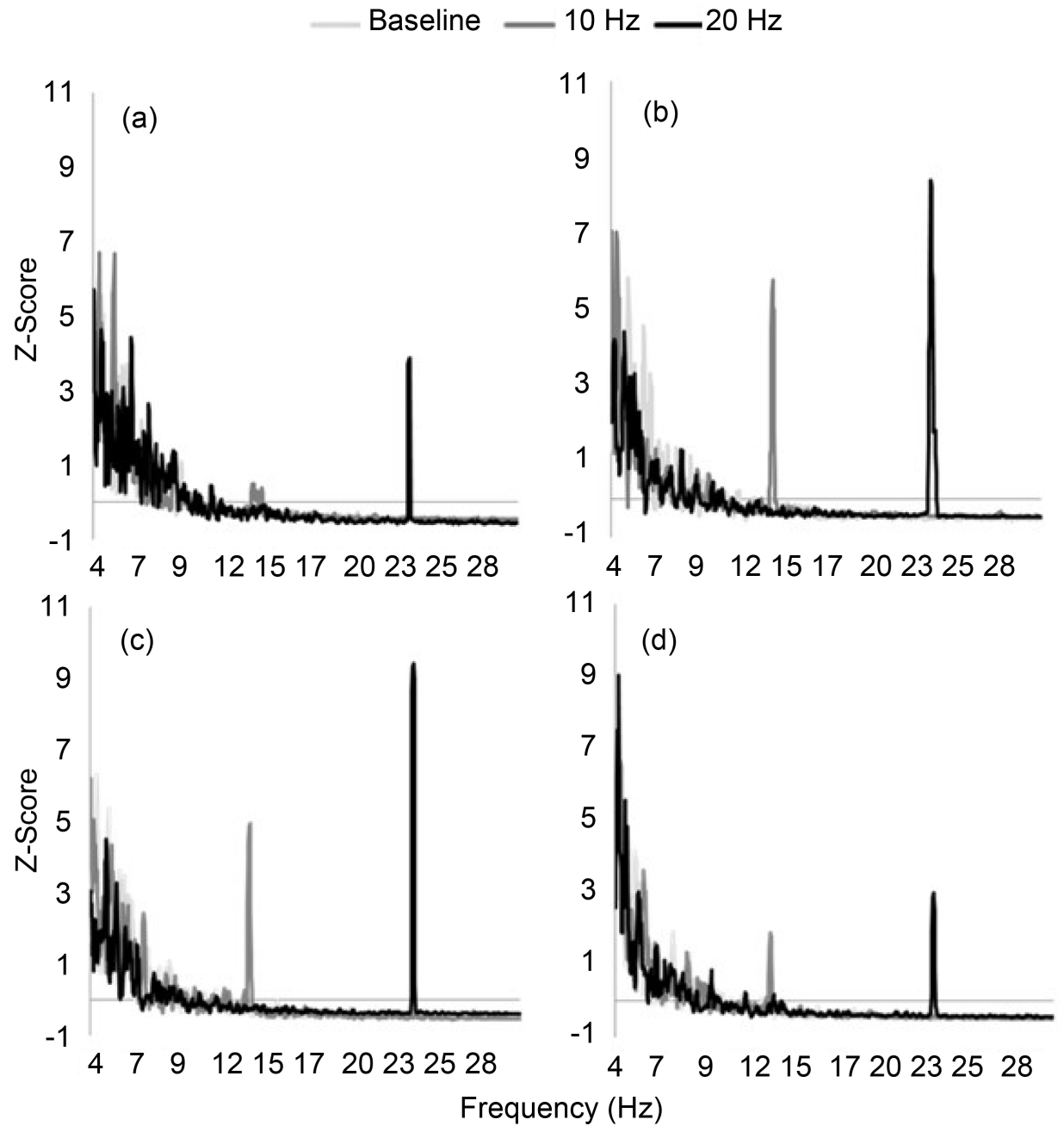

Figure 3. Standardized (Z-score) SPDs within the $4 \mathrm{~Hz}$ to $30 \mathrm{~Hz}$ range during baseline (light grey), $10 \mathrm{~Hz}$ (dark grey), and $20 \mathrm{~Hz}$ (black) light flash conditions for right (top) and left (bottom) optic nerve signal transductions measured at the level of the left (left) and right (right) occipital poles. Note that ipsilateral stimulation and measurement (b) \& (c) result in greater $\mathrm{z}$-scores $(\mathrm{z} \approx$ 9) than contralateral stimulation and measurement (a) \& (d) of $10 \mathrm{~Hz}$ and $20 \mathrm{~Hz}$ light flashes. 
that transduced signals frequencies were shifted positively by $\sim 3$ to 4 cycles per second $(\mathrm{Hz})$. These frequency shifts were reliable though not contingent upon frequency of input or hemisphere of expression.

\subsection{Frequency-Dependent Signal Processing}

An analysis of variance (ANOVA) revealed a phototransduction effect for global SPDs, $\mathrm{F}(5,269)=7.60, \mathrm{p}<0.001, \eta^{2}=0.13$. Narrow band effects were associated with similar degrees of explained variance: delta $\left(\eta^{2}=0.10\right)$, alpha $\left(\eta^{2}=0.11\right)$, betal $\left(\eta^{2}=0.12\right)$, beta2 $\left(\eta^{2}=0.10\right)$, and gamma $\left(\eta^{2}=0.05\right)$. Theta band effects were not observed $(\mathrm{p}>0.05)$. The primary source of variance was a difference within a computed average of alpha, beta1, and beta2 SPDs $(7.5 \mathrm{~Hz}-30 \mathrm{~Hz})$ between the "no phototransduction" condition $(\mathrm{M}=11.08, \mathrm{SEM}=0.13)$ and the right optic nerve phototransduction condition $(\mathrm{M}=$ 13.27, $\mathrm{SEM}=0.37), \mathrm{t}(178)=5.56, \mathrm{p}<0.001, \mathrm{r}^{2}=0.15$ is shown in Figure 4$)$. A less robust difference within the alpha-beta2 SPD range between the "no phototransduction" condition and left optic nerve phototransduction condition was noted $\left(\mathrm{r}^{2}=0.06\right)$, though both of these conditions did not differ from baseline alpha-beta2 SPDs ( $\mathrm{p}>$ 0.05). In other words, $7.5 \mathrm{~Hz}-30 \mathrm{~Hz}$ SPDs were identical whether or not white light was applied to the brain with or without photoresistor-mediated transduction with the exception of phototransduced signals which were carried through the right optic nerve (Figure 4).

Investigating stimulus-frequency-specific phototransduction mediated by the right optic nerve, alpha band $(7.5 \mathrm{~Hz}-14 \mathrm{~Hz})$ SPDs within the left occipital lobe increased during applied white light stimuli flashed at $3 \mathrm{~Hz}(\mathrm{M}=30.00, \mathrm{SEM}=2.85), 4 \mathrm{~Hz}(\mathrm{M}=$

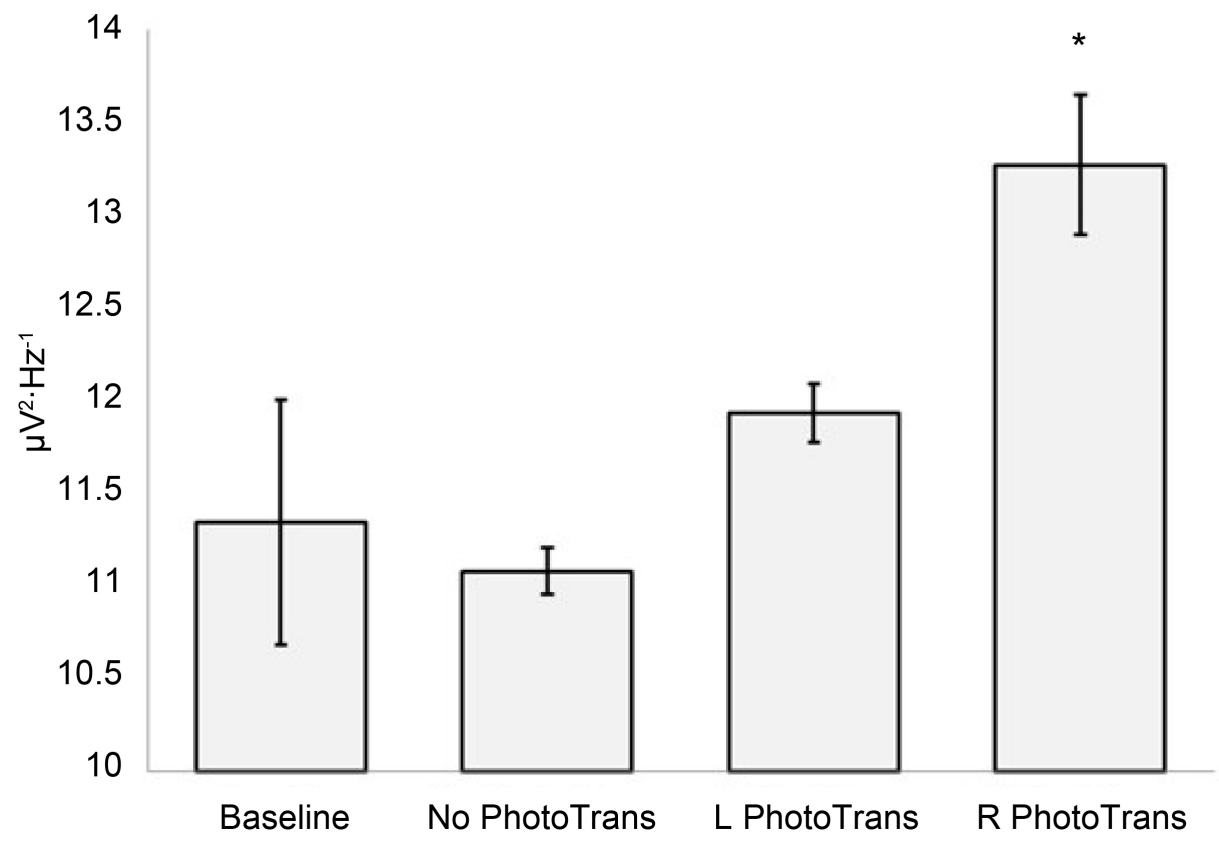

Figure 4. Averaged SPDs between $7.5 \mathrm{~Hz}$ and $30 \mathrm{~Hz}$ across experimental conditions: Baseline (no light stimulus, no photoresistor), No PhotoTrans (light, no photoresistor), L PhotoTrans (light, left optic nerve photoresistor), and R PhotoTrans (light, right optic nerve photoresistor). A significant difference from baseline was noted $(\mathrm{p}<0.001)$. 
23.53, $\mathrm{SEM}=0.55), 6 \mathrm{~Hz}(\mathrm{M}=29.50, \mathrm{SEM}=2.57), 30 \mathrm{~Hz}(\mathrm{M}=23.53, \mathrm{SEM}=0.55), 40$ $\mathrm{Hz}(\mathrm{M}=27.43, \mathrm{SEM}=0.89)$, and $70 \mathrm{~Hz}(\mathrm{M}=27.67, \mathrm{SEM}=1.88)$ relative to baseline. Effect sizes $\left(\mathrm{r}^{2}\right)$ associated with each of these significant differences were $0.69(3 \mathrm{~Hz})$, $0.86(4 \mathrm{~Hz}), 0.71(6 \mathrm{~Hz}), 0.70(30 \mathrm{~Hz}), 0.90(40 \mathrm{~Hz}), 0.73(70 \mathrm{~Hz})$ respectively. After Bonferroni correction ( $\alpha=0.008$ ), only effects associated with $4 \mathrm{~Hz}$ and $40 \mathrm{~Hz}$ remained statistically significant (Figure 5). Beta1 $(14 \mathrm{~Hz}-20 \mathrm{~Hz})$ and beta2 $(20 \mathrm{~Hz}-30$ $\mathrm{Hz}$ ) frequency bands did not demonstrate reliable departures from baseline SPD values $(\mathrm{p}>0.05)$.

The "no phototransduction" condition-which involved photostimulation of the brain without the insertion of a transducing photoresistor embedded within one of the optic nerves-was marked by an increase in delta band $(1.5 \mathrm{~Hz}-4 \mathrm{~Hz})$ SPDs within the right occipital pole relative to baseline when the frequency of the applied while light cycled at $60 \mathrm{~Hz}, \mathrm{t}(4)=6.10, \mathrm{p}<0.005, \mathrm{r}^{2}=0.90$ (Figure 6). These results were not entirely surprising as there is some evidence to suggest that microvolt potentials recorded within fixed brains change as a function of ambient light $(60 \mathrm{~Hz})$ exposure [11]. No other frequency of non-transduced photostimulation produced a statistically significant deviation in SPDs from baseline ( $\mathrm{p}>0.008)$.

\subsection{Photocell Array \& Dynamic Visual Images}

\subsubsection{Brodmann Area 17, Occipital Pole}

A reliable $\sim 200 \mu \mathrm{V}^{2} \cdot \mathrm{Hz}^{-1}$ decrease within the delta band was noted during the static white condition relative to the static black condition $\left(r^{2}=0.17\right)$, indicating simple light-dark discrimination. One-way ANOVAs revealed video type effects within the alpha-beta1 $(7.5 \mathrm{~Hz}-20 \mathrm{~Hz})$ band $\left(\eta^{2}=0.28\right)$ as well as for gamma SPDs $\left(\eta^{2}=0.28\right)$. Further investigation revealed the major sources of variance were SPDs within the alpha-beta1 band between a single moving light $(M=20.02, \mathrm{SEM}=2.75)$ and many moving lights $(\mathrm{M}=13.63, \mathrm{SEM}=0.50), \mathrm{t}(10)=2.28, \mathrm{p}<0.05$ (Figure 7 ). Together, these results suggested that upon complex stimulation of the right optic nerve electric

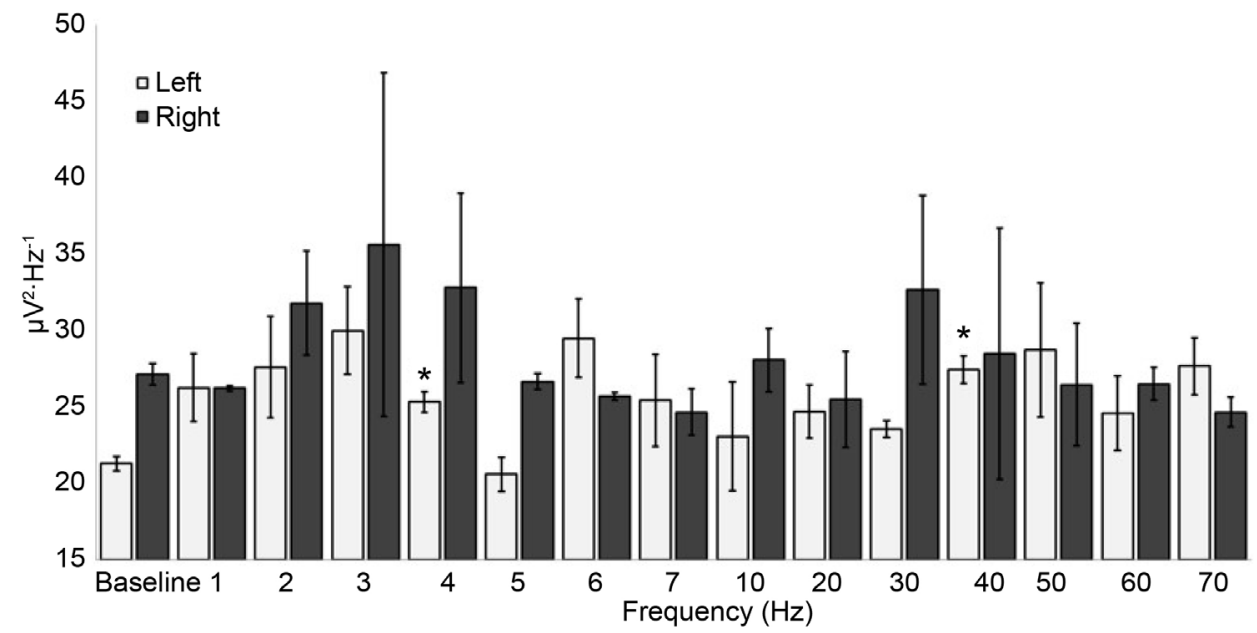

Figure 5. Alpha $(7.5 \mathrm{~Hz}-14 \mathrm{~Hz})$ SPDs within the left (light grey) and right (dark grey) occipital poles across frequencies of the applied photostimulation for conditions involving phototransduction by a photoresistor inserted into the right optic nerve. Significant differences from baseline after correction are noted $(\mathrm{p}<0.001)$. 


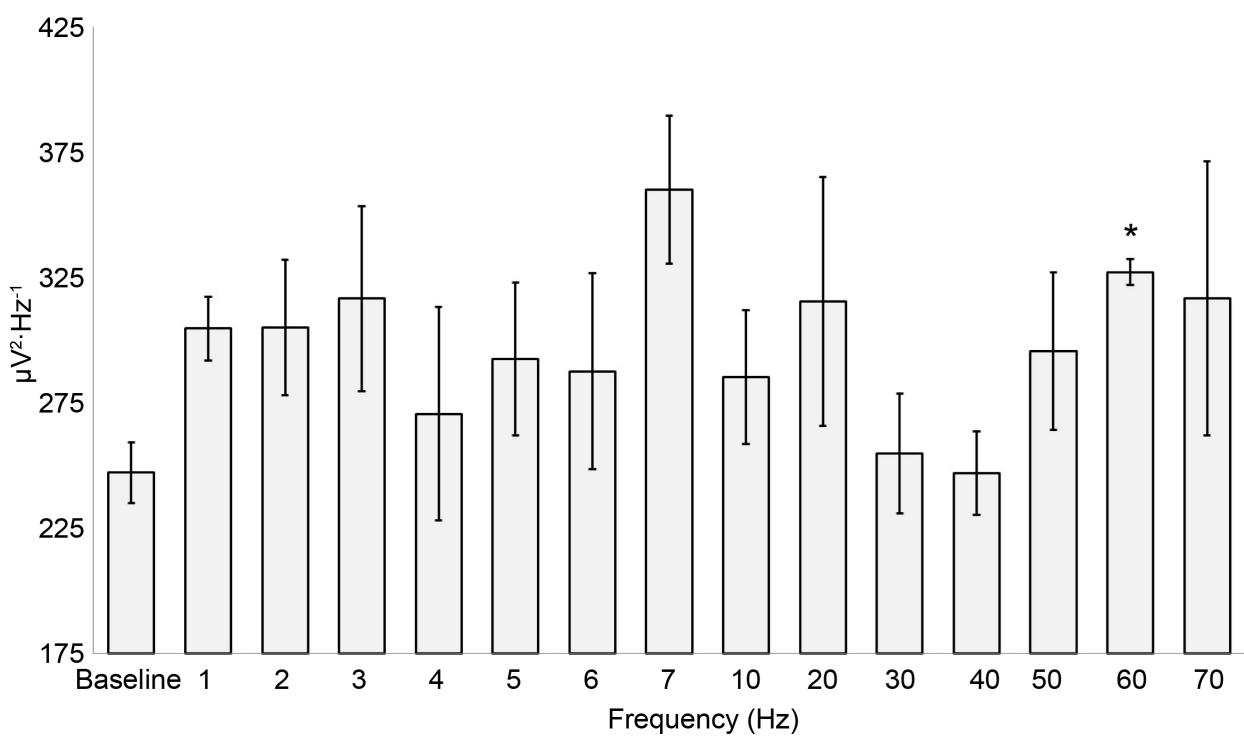

Figure 6. Delta $(1.5 \mathrm{~Hz}-4 \mathrm{~Hz})$ SPDs within the right occipital pole as a function of the frequency of the applied photostimulation for conditions not involving a photoresistor (i.e. no phototransduction). Significant differences from baseline are indicated $(\mathrm{p}<0.005)$.

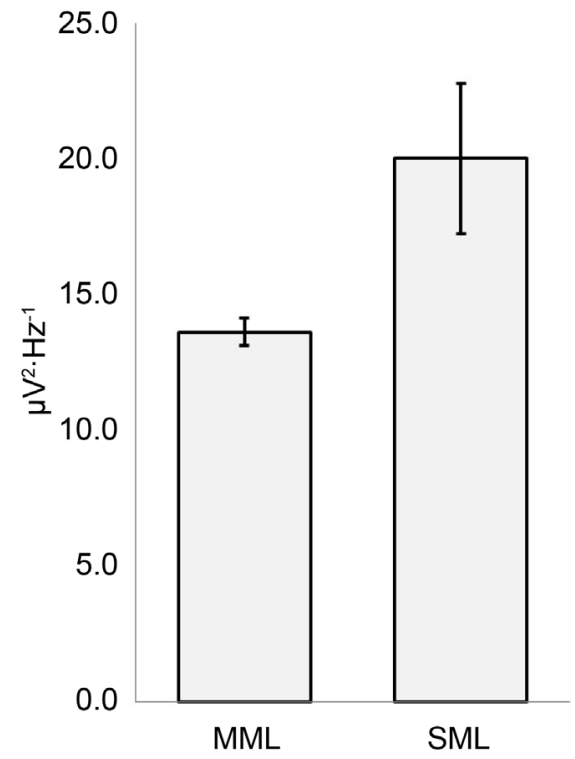

Figure 7. Alpha-betal $(7.5 \mathrm{~Hz}-20 \mathrm{~Hz})$ SPDs within the occipital polar area (BA17) dynamic visual conditions involving many moving lights (MML) and a single moving light (SML). The difference was statistically significant $(\mathrm{p}<0.05)$.

potential differences within BA17 displayed SPD discrimination indicative of first-order visual receptive processing-simple light-dark or contrast discrimination. Videos containing images normally associated with positive and negative affect were processed identically within BA17 of the unsectioned, fixed human brain ( $p>0.05)$. Mandelbrot fractals and other dynamic spatial configurations were not associated with SPD changes indicative of further processing capacity within this region. Mean power aggregates for video conditions containing movement did not induce SPD differences relative to conditions involving non-movement. 


\subsubsection{Dorsal Visual Stream}

ANOVAs revealed SPD differences within the theta $\left(\eta^{2}=0.58\right)$, alpha $\left(\eta^{2}=0.65\right)$, betal $\left(\eta^{2}=0.64\right)$, beta2 $\left(\eta^{2}=0.61\right)$, and gamma $\left(\eta^{2}=0.61\right)$ bands within the superior parietal region during different types of stimulation. Homogeneous subsets revealed that these differences were driven by frequency-independent $50-100 \mu \mathrm{V}^{2} \cdot \mathrm{Hz}^{-1}$ increases during the variable strobe condition relative to all others $(\mathrm{p}<0.001)$. SPD differences were not observed when comparing static white and black background conditions $(p>0.05)$. Excluding strobe conditions, increased gamma SPDs were identified during movement $(\mathrm{M}=1.31, \mathrm{SEM}=0.02)$ relative to non-movement $(\mathrm{M}=1.20, \mathrm{SEM}=0.02)$ video conditions, $\mathrm{t}(58)=2.76, \mathrm{p}<0.01, \mathrm{r}^{2}=0.12$. For the right superior parietal area, effects associated with movement were amplified for gamma SPDs, $\mathrm{t}(28)=2.76, \mathrm{p}=0.01, \mathrm{r}^{2}=0.21$. Additionally, increased alpha-betal SPDs were noted $\left(\mathrm{r}^{2}=0.44\right)$ during movement conditions relative to non-movement conditions (Figure 8). A discriminant analysis with varimax rotation loading alpha-betal and gamma SPDs for the right superior parietal area classified $80 \%$ of movement and non-movement cases in a corrected model $(\mathrm{n}=30), \Lambda=0.73, \chi^{2}(2)=8.65, \mathrm{p}<0.05$, canonical $\mathrm{R}^{2}=0.52$. When isolating the left superior parietal area, movement-associated SPD differences were abolished ( $p>0.05)$. Video conditions containing images typically associated with positive and negative affect did not induce relative SPD differences within the superior parietal area.

\section{Discussion}

The results demonstrate that the occipital and parietal cortices process signals differentially in a post-mortem human brain interfaced with arrays of photocells. We are not aware of any reported cerebrocortical functional observations in the literature with or without the assistance of an electronic interface. Throughout the history of neuroscientific study, unexpected properties of the human brain have been revealed by systematic investigation during specific contexts which were, at the time of discovery, considered not likely or impossible. Although the present study involved only a single brain, case demonstrations are historically not uncommon where the general operations of the

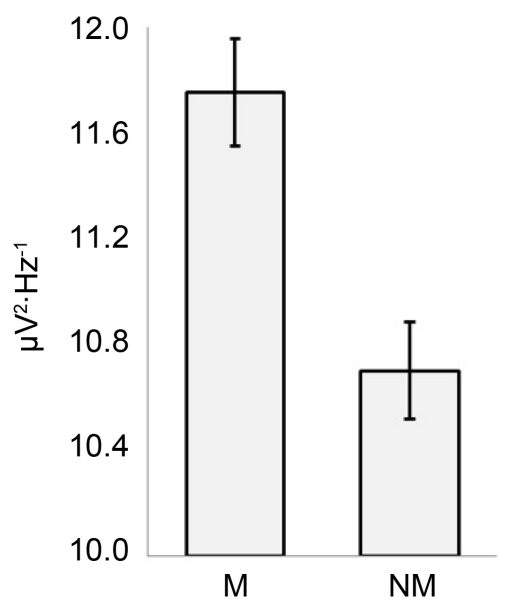

Figure 8. Alpha-betal $(7.5 \mathrm{~Hz}-20 \mathrm{~Hz})$ SPDs within the occipito-parietal area (dorsal visual stream) dynamic visual stimuli involving movement $(M)$ and non-movement (NM). The difference was statistically significant $(\mathrm{p}=0.001)$. 
human brain are typically revealed by representative individuals who underline key principles of neural science such as in the case of Broca [12] and Alzheimer [13]. These cases in particular ultimately defined entire fields of study. We did not have specific pre-mortem data for the brain that was measured in these experiments. Given this caveat, there was remarkable specificity to different photic stimulation through the optic nerve that in the balance of probabilities minimizes the possibility of artefact. The systematic relationship between the manipulated, experimental factors and the responses as inferred by spectral power differences was not indicative of noise or random processes. In addition, the laterality of the effect, the occurrence of changes only within specific frequency bands, and the differential responses of the primary visual cortices to simple light flashes compared to the adjacent parietal's response to movement in general (a spatial indicator) are consistent with the residual of function that is similar to that shown in the living brain.

We found that the specific frequency of the flashed light $(10 \mathrm{~Hz}$ or $20 \mathrm{~Hz})$ was reflected in an increased spectral power recorded from the occipital cortical regions. The effect was noted ipsilaterally for both right and left optical nerve stimulation and suggests that the temporal aspect of the optic nerve dominated the effect. Fedotchev et al. [14] showed that, in living subjects, the peaks in the spectral analyses of occipital electrical activity reflected the frequency of the flashes. Interestingly the power spectral associated with specific frequency flashes was not a narrow band effect but was distributed within a wider bin around the key frequency. The major difference between changing light frequency effects on brain activity is that the living brain usually shows blocking or suppression (particularly in alpha-band proportions). Although we did not observe "alpha blocking" during the presentation of light stimuli there was a significant decrease within the delta band during static white light compared to static black conditions. This suggests that the brain exhibited some variant of light-dark discrimination.

The fixed brain was not a passive reflector of the visual stimuli presented through the prosthetic retina. The primary visual cortices (area 17) displayed spatially different and frequency specific effects. The brain responded differentially to the different types of videos within the alpha-betal $(7.5 \mathrm{~Hz}$ to $20 \mathrm{~Hz})$ and gamma $(30 \mathrm{~Hz}$ to $40 \mathrm{~Hz})$ bands. In fact, there was a significant difference within the alpha-betal band when there was a single moving light compared to many moving lights. The complex videos containing emotional or non-emotional themes such as babies, dogs, or dancing humans did not elicit any specific changes within the primary visual cortices compared to the reference (non-movement) stimuli.

Within the region corresponding to the dorsal visual stream (BA 7 and 19), increased gamma activity, a presumed major correlate of complex perceptual processing, was measured if there was movement in the videos compared to non-movement. This was more evident in the right parietal region, though the absence of effects within the left hemisphere could be due to proportional contralateral signal loss. A discriminant analysis for the right superior parietal responses indicated that the differentiation between videos containing movement or no movement was due to the contributions from the alpha-beta 1 and gamma bands. The left parietal effect was negligible. Consequently, consistent with the functional asymmetry of the living human brain the right associa- 
tive visual (parietal) region displayed disproportional responsiveness to visual stimuli when they were moving.

We searched the literature and found no conspicuous experiments regarding this type of stimulation and measurement of the fixed, deceased human brain. We suspect that the technology such as photocells and sensitive quantitative EEG methods were not sufficiently sophisticated until recently to permit the type of discernment we have reported here. These results might be considered morbid or spurious to some; however, in the pursuit of understanding the relationship between cerebral functions and brain structure, assessment of the deceased, properly fixed human brains may reveal valuable information regarding the intrinsic structures upon which these complex phenomena are superimposed. The book that began modern molecular biology was appropriately titled by Schrodinger [15] in the form of a simple question: What is Life? The answer, in the form of inspired, independent experiments revealing that molecular machinery governs biological phenomena, has come to define modern medicine. Likewise, our question might be asked as follows: What is cognitive complexity and how much of that capacity can be maintained within the appropriately fixed post-mortem state?

\section{References}

[1] Rouleau, N., Lehman, B. and Persinger, M.A. (2016) Focal Attenuation of Specific Electroencephalographic Power over the Right Parahippocampal Region during Transcerebral Copper Screening in Living Subjects and Hemispheric Asymmetric Voltages in Fixed Coronal Sections. Brain Research, 1644, 267-277. https://doi.org/10.1016/j.brainres.2016.05.034

[2] Rouleau, N. and Persinger, M.A. (2016) Differential Responsiveness of the Right Parahippocampal Region to Electrical Stimulation in Fixed Human Brains: Implications for Historical Surgical Stimulation Studies? Epilepsy \& Behaviour, 60, 81-186. https://doi.org/10.1016/j.yebeh.2016.04.028

[3] Diamond, M.C., Scheibel, A.B., Murphy, G.M. and Harvey, T. (1985) On the Brain of a Scientist: Albert Einstein. Experimental Neurology, 88, 198-204. https://doi.org/10.1016/0014-4886(85)90123-2

[4] Witelson, S.F., Kigar, D.L. and Harvey, T. (1999) The Exceptional Brain of Albert Einstein. The Lancet, 353, 2149-2153. https://doi.org/10.1016/S0140-6736(98)10327-6

[5] Fritsch, G. and Hitzig, E. (1870) Über die elektrische Erregbarkeit des Grosshirns. von Bonin, G., Trans. In: The Cerebral Cortex, Thomas, Springfield, IL, 73-96.

[6] Thomas, R.K. and Young, C.D. (1993) A Note on the Early History of Electrical Stimulation of the Human Brain. The Journal of General Psychology, 120, 73-81. https://doi.org/10.1080/00221309.1993.9917863

[7] Krause, F. (1912) Surgery of the Brain and Spinal Cord Based on Personal Experience. Haubold, A. and Thorek, M., Trans. Rebman, New York, NY.

[8] Penfield, W. and Boldrey, E. (1937) Somatic Motor and Sensory Representation in the Cerebral Cortex of Man as Studied by Electrical Stimulation. Brain: A Journal of Neurology, 60, 389-443. https://doi.org/10.1093/brain/60.4.389

[9] Penfield, W. and Welch, K. (1951) The Supplementary Motor Area of the Cerebral Cortex: A Clinical and Experimental Study. AMA Archives of Neurology \& Psychiatry, 66, 289-317. https://doi.org/10.1001/archneurpsyc.1951.02320090038004

[10] Crosby, E.C., Humphrey, T. and Lauer, E.W. (1962) Correlative Anatomy of the Nervous System. Chapter 7: Telencephalon, Part I-Gross Structure of the Telencephalon. The 
Macmillan Company, New York, 343-355.

[11] Costa, J., Rouleau, N. and Persinger, M.A. (2016) Differential Spontaneous Photon Emissions from Cerebral Hemispheres of Fixed Human Brains: Asymmetric Coupling to Geomagnetic Activity and Potentials for Examining Post-Mortem Intrinsic Photon Information. Neuroscience \& Medicine, 7, 49-59. https://doi.org/10.4236/nm.2016.72006

[12] Broca, P.P. (1861) Loss of Speech, Chronic Softening, and Partial Destruction of the Anterior Left Lobe of the Brain. Bulletin de la Société Anthropologique, 2, 235-238.

[13] Alzheimer, A. (1907) About a Peculiar Disease of the Cerebral Cortex. Allgemeine Zeitschrift fur Psychiatrie und Psychish-Gerichtlich Medicin, 64, 146-148.

[14] Fedotchev, A.I., Bondar, A.T. and Konovalov, V.F. (1990) Stability of Resonance EEG Reactions to Flickering Light in Humans. International Journal of Psychophysiology, 9, 189-193. https://doi.org/10.1016/0167-8760(90)90073-M

[15] Schrödinger, I. (1944) What Is Life? Cambridge University Press, London, UK.

Submit or recommend next manuscript to SCIRP and we will provide best service for you:

Accepting pre-submission inquiries through Email, Facebook, LinkedIn, Twitter, etc. A wide selection of journals (inclusive of 9 subjects, more than 200 journals)

Providing 24-hour high-quality service

User-friendly online submission system

Fair and swift peer-review system

Efficient typesetting and proofreading procedure

Display of the result of downloads and visits, as well as the number of cited articles

Maximum dissemination of your research work

Submit your manuscript at: http://papersubmission.scirp.org/

Or contact ojbiphy@scirp.org 